\title{
Development of a CiFT Co-expression System for Functional Analysis of Genes in Citrus Flowers and Fruit
}

\author{
Tomoko Endo $^{1 *}$, Takehiko Shimada ${ }^{1}$, Hiroshi Fujii ${ }^{1}$, Fumie Nishikawa1, Aiko Sugiyama ${ }^{2}$, \\ Michiharu Nakano², Tokurou Shimizu ${ }^{1}$, Yasushi Kobayashi ${ }^{3}$, Takashi Araki ${ }^{4}$, \\ Leandro Peña ${ }^{5}$ and Mitsuo Omura ${ }^{2}$
}

\author{
${ }^{1}$ National Institute of Fruit Tree Science, National Agricultural Research Organization, Okitsunakachou Shimizu-ku, Shizuoka 424- \\ 0292, Japan \\ ${ }^{2}$ Faculty of Agriculture, Shizuoka University, Ohya, Suruga-ku, Shizuoka 422-8529, Japan \\ ${ }^{3}$ Max Planck Institute for Developmental Biology, D-72076 Tübingen, Germany \\ ${ }^{4}$ Division of Integrated Life Science, Graduate School of Biostudies, Kyoto University, Sakyo-ku, Kyoto 606-8501, Japan \\ ${ }^{5}$ Instituto Valenciano de Investigaciones Agrarias (IVIA), Apartado Oficial, 46113-Moncada, Valencia, Spain
}

\begin{abstract}
We have developed a CiFT co-expression system for the rapid evaluation of transgenic citrus flowers and fruit. In order to co-express a target gene and the $C i F T$ gene, a $C i F T$ co-expression vector was constructed by inserting the fragment containing a chimeric target gene into the binary vector harboring P35S::CiFT. This system was applied to metabolically engineer aroma components in trifoliate orange (Poncirus trifoliata L. Raf). In order to reduce the limonene level, a gene encoding limonene synthase (CitMTSE1) from satsuma mandarin (Citrus unshiu Marc.) was introduced into trifoliate orange in an anti-sense orientation with the $C i F T$ co-expression vector. Transgenic plants flowered extremely early, and began to produce normal fruit within 2 years of Agrobacterium infection. Transcripts of transgenes were accumulated in reproductive tissues of transgenic plants, and endogenous transcripts for limonene synthase were reduced. The ratio of limonene to other monoterpenes was significantly decreased in flowers and fruit of transgenic plants. These results clearly indicated that the CiFT coexpression system is a useful tool for the functional analysis of genes in citrus flowers and fruit. Such analyses are important for several applications, including the metabolic engineering of aroma components in citrus.
\end{abstract}

Key Words: CiFT, citrus, co-expression, limonene, metabolic engineering.

\section{Introduction}

Citrus, one of the most commercially important fruit crops, has many unique characteristics, such as various secondary metabolites, parthenocarpy, seedlessness, apomixes, and sexual incompatibility. However, the long juvenile period, ranging from 6 to 20 years, has hampered genetic studies on these characteristics, as well as traditional breeding. For example, a recent Japanese citrus cultivar, 'Harehime', was released in 2001, originating from a cross in 1990. It took about 5 years to first fruiting in spite considerable efforts to shorten the juvenile period by top-grafting (Yoshida et al., 2005). Reduction of the generation time in citrus would

Recieved; March 21, 2008. Accepted; July 25, 2008.

* Corresponding author (E-mail: tomoen@affrc.go.jp). accelerate studies on the complex regulatory mechanisms that underlie biological and agronomical traits, and lead to the manipulation of agronomically important traits, such as the nutritional value and fruit quality. Recently, a reduction of the long juvenile period of citrus has been achieved using flowering genes such as APETALA1 (AP1), LEAFY (LFY), and the Citrus homolog of FLOWERING LOCUS T (CiFT) (Endo et al., 2005; Peña et al., 2001). The overexpression of either of these genes caused early flowering and fruiting within a few years of Agrobacterium infection. In recent years, the Arabidopsis FT gene and its orthologous gene in rice, $H d 3 a$, have emerged as the most plausible candidates for the flower-inducing hormone "florigen". FT and Hd3a proteins are produced in leaves, move to shoot apices, and then induce flowering (Abe et al., 2005; Corbesier et al., 2007; Takada and Goto, 2003; Tamaki 
et al., 2007). Therefore, CiFT is one of the most useful genes to develop new experimental systems to reduce the time until flowering and fruiting in citrus transgenic plants, and to rapidly evaluate the effects of introduced genes on these reproductive tissues. Such experimental tools will advance functional analyses of various genes related to morphogenesis and fruit quality in citrus.

The flavors in Citrus and the closely related genera Poncirus and Fortunella are characterized by a variety of monoterpenes and sesquiterpenes (Sawamura, 2000). A typical feature of the volatile substances in most citrus fruit is that $d$-limonene comprises more than $70 \%$ of the essential oils. The high proportion of $d$-limonene in volatile citrus substances is thought to result from the lower $\mathrm{Km}$ value of $d$-limonene synthase compared with other monoterpene synthases (Lücker et al., 2002). Several monoterpene synthases have been isolated from citrus species (Lücker et al., 2002; Shimada et al., 2004, 2005a, 2005b), and different volatile compositions in flowers and fruit were explained by different patterns of transcript accumulation of the corresponding monoterpene synthase genes (Shimada et al., 2005a). So far, metabolic engineering to modify monoterpene biosynthesis has been attempted in tomato (Lewinsohn et al., 2001), petunia (Lücker et al., 2001), and tobacco (Lücker et al., 2004a, 2004b; Ohara et al., 2003). However, their corresponding products in transgenic plants accumulated at very low levels, or were inactivated by glycosylation and hydroxylation. Overexpression of the geraniol synthase (GES) gene in mature tomato fruit caused marked changes in the volatile composition, along with unexpected increases of myrcene, limonene, and ocimene (Davidovich-Rikanati et al., 2007). Given these very different results in different plant species, it will be of interest to determine the effects of modifying the transcription of monoterpene synthase genes on aroma components in citrus flowers and fruit.

In this study, we developed a new experimental system - the CiFT co-expression system - in which a target gene was co-introduced and co-expressed with CiFT in transgenic plants. This system was applied to metabolic engineering for aroma components in trifoliate orange (Poncirus trifoliata L. Raf.). The anti-sense sequence of a gene encoding $d$-limonene synthase (CitMTSE1) was introduced along with P35S::CiFT to reduce limonene levels in the flowers and fruit of trifoliate orange. Transgenic plants which developed flowers and fruit were obtained within 2 years of Agrobacterium infection. Gene expression and the composition of volatiles were analyzed in transgenic plants, and we observed a significant reduction of limonene levels compared with control plants.

\section{Materials and Methods}

\section{Plant materials and vector construction}

Trifoliate orange (Poncirus trifoliata L. Raf), sour orange (Citrus aurantium L.), and rough lemon (Citrus jambhiri Lush.) were used in these experiments. Plants were cultivated at the National Institute of Fruit Tree Science (NIFTS), Okitsu Citrus Research Station, Shizuoka, Japan. Trifoliate orange was used as the source for transformation experiments. Sour orange was used as the first rootstock for the in vitro grafting of regenerated shoots. Rough lemon was used as the second rootstock for the second grafting of trifoliate orange on sour orange. Seeds from all species were germinated and grown as described previously (Endo et al., 2005) to obtain the materials required for the experiments.

A shuttle vector (Fig. 1a) was constructed as follows: The binary vector pBE2113 (Mitsuhara et al., 1996) was digested with $X b a \mathrm{I}$ and $S a c \mathrm{I}$ to remove the uidA fragment, and ligated with the linker fragment obtained by the digestion of pUC18 (Yanisch-Perron et al., 1985) with $\mathrm{XbaI}$ and $\mathrm{SacI}$. The resultant vector was used as a template for a PCR reaction with a set of primers: pBI101F2419: 5'-CTTCCGGCTCGTATGTTGTGT-3' and pBI101R4775H: 5'-ATAAGCTTGGCGAAAGGG GGATGTGCT-3'; HindIII site underlined. The amplified fragment contained the $E l_{2}-35 S$ region, which comprises $\mathrm{El}_{2}$ (duplicated 5'-upstream (-419 to -90 ) sequence of cauliflower mosaic virus (CaMV) $35 \mathrm{~S}$ promoter), the 5 --upstream ( -90 to -1$)$ sequence of the CaMV 35S promoter and 5'-untranslated $(\Omega)$ sequence of tobacco mosaic virus (TMV), and the nopaline synthase terminator (Tnos). It was inserted into the HindIII site of a modified pUC18 that lacked the cloning sites from $S p h \mathrm{I}$ to $E c o$ RI. The $C i F T$ co-expression vector was constructed using this shuttle vector. The $d$-limonene synthase 1 (CitMTSE1) cDNA clone (AB110636) from satsuma mandarin (C. unshiu Marc.) (Shimada et al., 2004) was amplified with TAEASY1F-Xba (5'-AT TCTAGATCATCCTTTGGTGACAGGTG-3'; XbaI site underlined) and TAEASY1R-Sma (5'-ATCCCGGG ATGTCTTCTTGCATTAATCC-3'; SmaI site underlined), digested, and inserted downstream of $E l_{2} 35 \mathrm{~S}$ on the shuttle vector with an anti-sense orientation (Fig. 1b). This construct was digested with HindIII, and the chimeric gene was inserted into the binary vector pCGN1547 (Calgene, USA) harboring P35S::CiFT (Kobayashi et al., 1999). The CiFT co-expression vector (Fig. 1c) was transferred into the Agrobacterium tumefaciens strain LBA4404 by triparental mating.

\section{Agrobacterium-mediated transformation}

The transformation method for trifoliate orange was as described previously (Endo et al., 2005). Adventitious shoots emerging from segments were detached and individually subcultured on MS medium supplemented with $3 \%$ sucrose, $10^{-7} \mathrm{M}$ NAA, $10^{-8} \mathrm{M} \mathrm{GA}_{3}$, and $0.2 \%$ gelrite ( $\mathrm{pH}$ 5.6) for 1 to 3 months. Shoots were then grafted in vitro onto the etiolated seedlings of sour orange, which had been previously germinated and prepared as rootstock. In vitro-grafted plants were grafted again onto rough lemon rootstock and grown in 
(a)

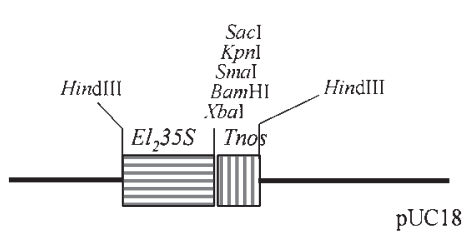

(b)

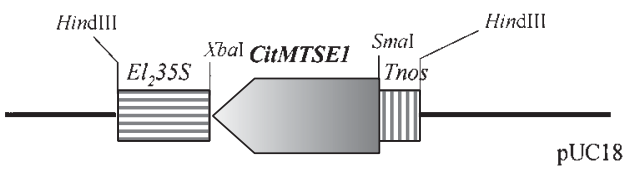

(c)

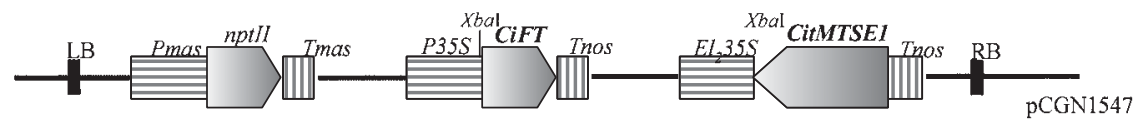

Fig. 1. Structures of shuttle vector (a), shuttle vector with anti-sense CitMTSE1 (b), and CiFT co-expression vector (c). The target gene, in this case the anti-sense CitMTSE 1, is finally inserted into the pCGN1547 binary vector harboring P35S::CiFT through the shuttle vector. LB, left border of T-DNA; RB, right border of T-DNA; $P 35 \mathrm{~S}$, Cauliflower mosaic virus $35 \mathrm{~S}$ promoter; $\mathrm{El}_{2}-35 \mathrm{~S}, \mathrm{El}_{2}$ (duplicated 5 '-upstream $(-419$ to -90$)$ sequence of $P 35 S)-5$ '-upstream $(-90$ to -1$)$ sequence of $P 35 S-5$ '-untranslated $(\Omega)$ sequence of TMV from pBE2113; Pmas, mannopine synthase promoter; Tnos, nopaline synthase terminator; Tmas, mannopine synthase terminator; npt II, kanamycin resistance gene.

a greenhouse. Control plants free of infection were concurrently regenerated and grown without kanamycin selection.

Gene incorporation was confirmed in transgenic plants by PCR, using three sets of primers: P35S (5'ATCTCCACTGACGTAAGGGATGACG-3') and Tnos (5'-ACAGGATTCAATCTTAAGAAACTTT-3'); P35S and CiFTgR4 (5'-AAAGCTGGCCCCTGTGGTTGC3'); P35S and TAE1-R1 (5'-AGAAACTGAGCTTTGC GAGGGAC-3').

\section{Southern blot analysis}

Transgenic trifoliate orange genomic DNA was extracted from fresh, fully expanded leaves according to Dellaporta et al. (1983). DNA was quantified using a NanoDrop ND-1000 spectrophotometer (NanoDrop Technologies, USA). To estimate the number of transferred genes in transgenic plants, $10 \mu \mathrm{g}$ of total DNA was completely digested with $X b a \mathrm{I}$ and analyzed by Southern blot. A $0.87 \mathrm{~kb}$ fragment obtained from the digestion of pBI221 (BD Biosciences, USA) with EcoRV and HindIII, which contained the $P 35 S$ enhancer region, was used as a probe. Digested DNA was separated by electrophoresis on a $1.0 \%(\mathrm{v} / \mathrm{v})$ agarose gel and blotted onto a nylon membrane (Hybond-NX, GE Healthcare, UK). Probe labeling by digoxigenin (DIG), hybridization, and detection were conducted according to the manufacturer's instructions (Roche Diagnostics GmbH, Germany). Washing was carried out twice in $0.1 \times$ SSC and $0.1 \% \mathrm{SDS}$ at $68^{\circ} \mathrm{C}$ for $15 \mathrm{~min}$, and the membrane was then exposed to X-ray film.
Gene expression analysis by reverse transcriptionpolymerase chain reaction ( $R T-P C R$ )

Total RNA was extracted from the leaves, flowers, juice sacs, and peel of mature fruit of transgenic plants using the methods described by Ikoma et al. (1996). RNA was quantified using a NanoDrop ND-1000 spectrophotometer. For RT-PCR, the first strand cDNA was synthesized from $1 \mu \mathrm{g}$ of total RNA by reverse transcriptase with Oligo-(dT) primers according to the instructions of the Ready-To-Go You-Primed First Strand Kit (GE Healthcare). The first strand cDNA was diluted to $60 \mu \mathrm{L}$ in water, and aliquots of $1 \mu \mathrm{L}$ were used in each RT-PCR reaction (total volume: $20 \mu \mathrm{L}$ ). PCR reactions were performed in a thermal cycler under the following conditions: 24 cycles of $10 \mathrm{~s}$ at $94^{\circ} \mathrm{C}, 15 \mathrm{~s}$ at $58^{\circ} \mathrm{C}$, and $90 \mathrm{sec}$ at $72^{\circ} \mathrm{C}$. The gene expressions of $\mathrm{CiFT}$, the endogenous limonene synthase gene in trifoliate orange (PtLim1), and total limonene synthase genes (introduced CitMTSE1 and endogenous PtLiml) were analyzed with the following primer sets: CiFT (5'GCTTAGTGTTGTTGAGTGTTTGTTTG-3' and 5'AAAGCTGGCCCCTGTGGTTGC-3'); PtLim1 (5'TGTTTTCTTGCGCTTTAC-3' and 5'-GTCGAGGCCA ACATCAATCGAAACTG-3'); total limonene synthase genes (5'-ATGTCTTCTTGCATTAATCCCTCA-3' and 5'-ATGCTACCAAGCTGTCCCTCGCA-3'). The full length of PtLim 1 was isolated using the SMART RACE cDNA Amplification Kit (Clontech, USA) and the sequence of its 3' UTR region was utilized to design specific primers for PtLim1. The accession number of PtLim1 is AB363936. The primers designed to amplify the alpha subunit of the elongation factor $1(\mathrm{EF} 1-\alpha)$ gene 
(5'-AAGGCTGAGCGTGAACGTGG-3' and 5'-ACG GCAATGTGGGAGGTGTG-3') were used for internal control reactions. The PCR products of each reaction were analyzed by electrophoresis on $1.5 \%(\mathrm{v} / \mathrm{v})$ agarose gels.

Extraction of volatile components and GC-MS analysis

Flowers at anthesis and the peel of mature fruit were collected from transgenic plants and prepared for the analysis of their volatile components according to the method of Hara et al. (1999). The instrument and assay conditions for GC-MS analysis were as follows: instrument, MSD 5890 (Hewlett Packard, USA); HP-1 capillary column, $30 \mathrm{~m} \times 0.25 \mathrm{~mm}$ internal diameter; film thickness, $2.5 \mu \mathrm{m}$, (Agilent Technologies, USA); carrier gas, He $\left(1.0 \mathrm{ml} \cdot \mathrm{min}^{-1}\right)$; injector temperature, $100^{\circ} \mathrm{C}$; ionization voltage, EI $70 \mathrm{eV}$; oven program, started at $50^{\circ} \mathrm{C}$ (5-min hold), $5^{\circ} \mathrm{C} \cdot \mathrm{min}^{-1}$ to $200^{\circ} \mathrm{C}$.

\section{Results}

Construction of CiFT co-expression vector and production of transgenic plants

In order to drive a target gene with the CiFT gene, we developed a CiFT co-expression system (Fig. 1). The target gene was inserted into the binary vector harboring P35S::CiFT (Kobayashi et al., 1999) through the shuttle vector. The shuttle vector has the $E l_{2}-35 S$ region (see Materials and Methods) and the nopaline synthase terminator (Tnos) of pBE2113 (Mitsuhara et al., 1996) (Fig. 1a). The $d$-limonene synthase 1 gene (CitMTSE1) was inserted with an antisense orientation between the promoter and terminator of this shuttle vector (Fig. 1b). The chimeric gene $\left(E l_{2}-35\right.$ S:: anti-CitMTSE1::Tnos) was inserted into the HindIII site of the binary vector harboring P35S: :CiFT (Kobayashi et al., 1999; Fig. 1c). The binary vector, which had $P 35 S: \because C i F T$ and a target gene, was designated as the CiFT co-expression vector.

A total of 494 epicotyl segments from etiolated seedlings of trifoliate orange were infected with A.tumefaciens LBA4404 harboring the CiFT coexpression vector. Adventitious shoots that regenerated from the segments on the selection medium were excised individually and grafted twice to promote growth (see Materials and Methods). After the second grafting, regenerated plants were transferred to the greenhouse. PCR analysis showed the presence of the transgene in 16 regenerated plants.

Southern blot analysis was carried out to confirm the integration of the transgene into the trifoliate orange genome, and to estimate the transgene copy number. Genomic DNA of each transgenic plant was digested with $X b a \mathrm{I}$ and subjected to hybridization with a DIGlabeled $P 35 S$ probe. All plants had the expected $1.7 \mathrm{~kb}$ fragment derived from the region between two $\mathrm{XbaI}$ sites in the T-DNA region (Figs. 1c and Fig. 2). The signals except for $1.7 \mathrm{~kb}$ were thought to show line-dependent fragments between the $X b a \mathrm{I}$ site downstream of P35S in the T-DNA and the $X b a \mathrm{I}$ site in the genomic sequence flanked with the left border. The fragment pattern showed that 16 transgenic plants were derived from 14 independent transgenic lines. A single copy of the transgene was detected in 9 lines, and two copies were detected in 5 lines.

Transgenic plants began to flower as early as 30 weeks after Agrobacterium infection, just after transfer to the greenhouse. Corresponding to the previous observation (Endo et al., 2005), the flowering of transgenic plants occurred continually. Some transgenic plants started fruit

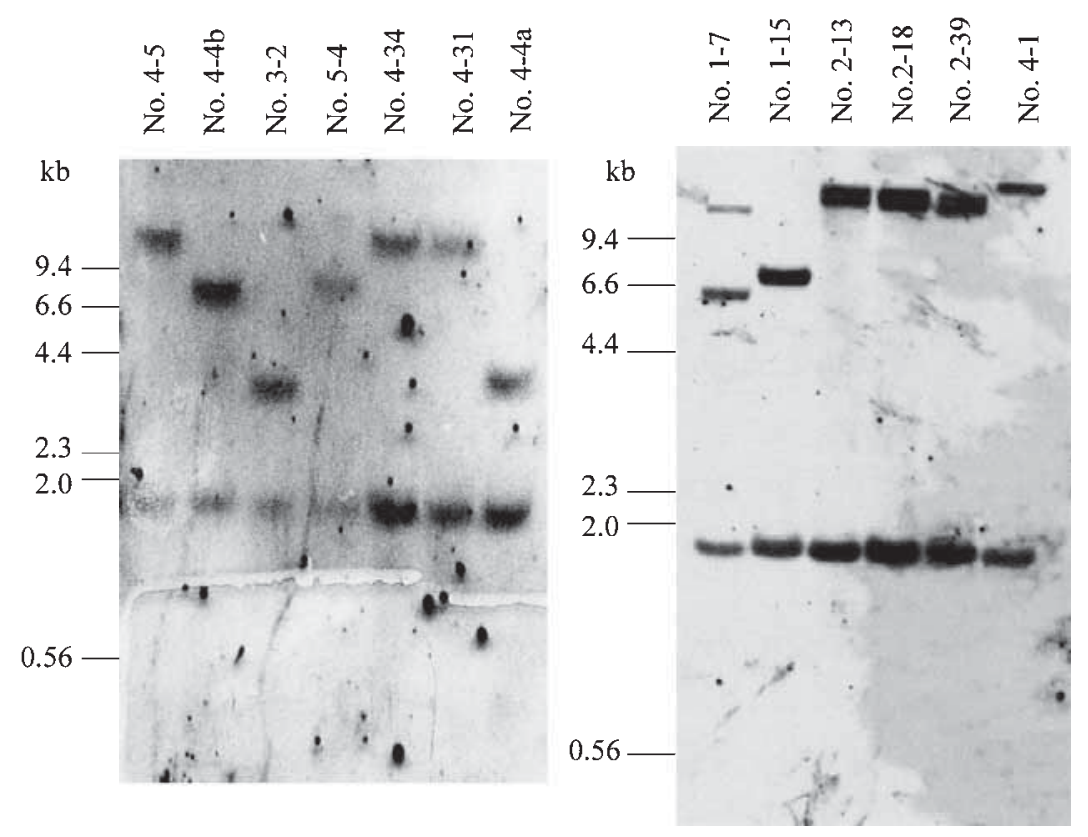

Fig. 2. Southern blot analysis of transgenic plants. Genomic DNA $(10 \mu \mathrm{g})$ from each transgenic plant was digested with $X b a \mathrm{I}$ and hybridized with a fragment of the $P 35 S$ enhancer region. 
development as early as 1 year after Agrobacterium infection. Within 2 years of Agrobacterium infection, all transgenic lines developed morphologically normal flowers (Fig. 3a) and six lines began to develop morphologically normal fruit (Fig. 3b). This was the same phenotype as the transgenic trifoliate orange with $P 35 S:: C i F T$ in the previous report (Endo et al., 2005). The efficiency of obtaining transgenic lines with flowers or fruit was approximately 2.8 or $1.2 \%$, respectively, based on the number of epicotyl segments infected. Six independent transgenic lines (1-7, 1-15, 2-39, 4-1, 4-34, and 5-4) were used for further analyses (Table 1).

\section{Gene expression analysis in transgenic plants}

Prior to gene expression analysis in transgenic plants, we isolated the endogenous limonene synthase gene in trifoliate orange and the full-length nucleotide and amino acid sequences were registered as PtLim 1 in DDBJ (AB363936). PtLim 1 consists of 2,133 bp with an ORF of $1,824 \mathrm{bp}$. It shows $96.0 \%$ sequence homology with the ORF of CitMTSE1 at the nucleotide sequence level. In order to discriminate endogenous gene expression from that of the transgene, a PtLim 1-specific primer set was designed from the 3' UTR sequence, which showed a low-level similarity with the introduced CitMTSE1. In the wild-type trifoliate orange, the transcript of PtLim 1 accumulated in the flowers and peel, but did not accumulate in leaves, stems, and juice sacs (Fig. 4). This is similar to the gene expression pattern of CitMTSE1

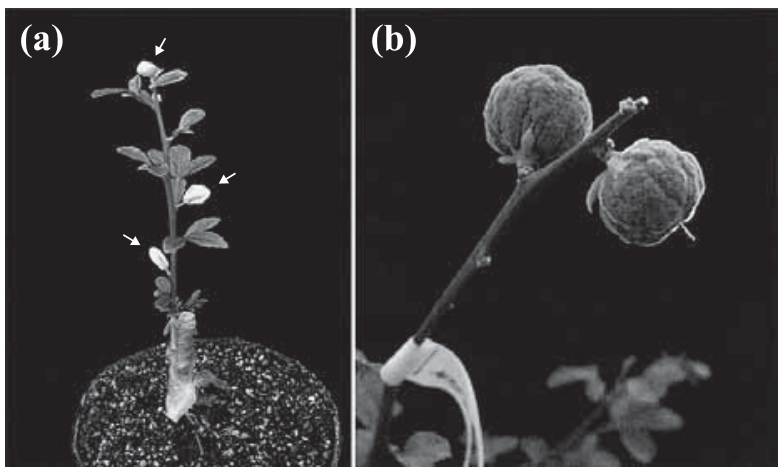

Fig. 3. Flowers (a: arrow) and fruit (b) of transgenic trifoliate orange (line No. 5-4).

Table 1. Summary of transgenic plants further analyzed.

\begin{tabular}{lccc}
\hline \hline Line & $\begin{array}{c}\text { Time to flowering } \\
\text { (month) }\end{array}$ & $\begin{array}{c}\text { Fruit } \\
\text { development }\end{array}$ & $\begin{array}{c}\text { Estimated copy No. } \\
\text { of transgenes }\end{array}$ \\
\hline $1-7$ & 8 & $+^{\mathrm{y}}$ & 2 \\
$1-15$ & 7 & - & 1 \\
$2-39$ & 12 & + & 2 \\
$4-1$ & 9 & - & 1 \\
$4-34$ & 14 & + & 1 \\
$5-4$ & 7 & + & 1 \\
\hline
\end{tabular}

z Observation after Agrobacterium infection.

${ }^{y}$ Presence (+) or absence (-) of fruit development and maturation on the plant. in satsuma mandarin, except that satsuma mandarin accumulates transcripts in juice sacs at a mature stage (Shimada et al., 2004, 2005b).

For transgenic plants, transcript levels of CiFT, total limonene synthase genes (introduced CitMTSE1 and endogenous PtLim1), and endogenous PtLim1 were investigated in leaves, flowers, and mature fruit by RTPCR. Since the fruit of transgenic Nos. 4-1 and 1-15 dropped before maturation, gene expression analysis of fruit was not carried out for these two lines. Since the primer set for CitMTSE1 amplified both the transgene and endogenous genes, gene expression of the introduced CitMTSE1 was deduced from the difference between the expression patterns of PtLiml and total limonene synthase genes (introduced CitMTSE1 and endogenous PtLim1). The CiFT transcript was detected in all tissues examined (Fig. 5). Transcripts of total limonene synthase genes (introduced CitMTSE1 and endogenous PtLim 1) were also detected in all tissues examined (Fig. 5). The PtLim1 transcript was not detected in leaves of any transgenic lines (Fig. 5a). This agreed with the wild-type result (Fig.4) and indicated that all the transcripts for total limonene synthase genes in transgenic leaves were attributable to the transgene. The PtLim1 transcript was detected in flowers of transgenic lines Nos. 1-7 and 239 , and slightly in No. 4-1, but barely in Nos. 4-34, 115, and 5-4 (Fig. 5b). In fruit, the PtLim1 transcript was detected in peel of Nos. 1-7 and 2-39 but barely in Nos. 4-34 and 5-4 (Fig. 5c). These results indicated that, at least in some transgenic lines such as Nos. 4-34 and 54, anti-sense transcripts for the introduced CitMTSE1 accumulated in all tissues, and expression levels of the endogenous limonene synthase gene in flowers and fruit were reduced. Therefore, it was confirmed that the introduction of the CiFT co-expression vector caused ectopic expression both of CiFT and anti-sense CitMTSE1 in tissues including flowers and fruit of transgenic plants.

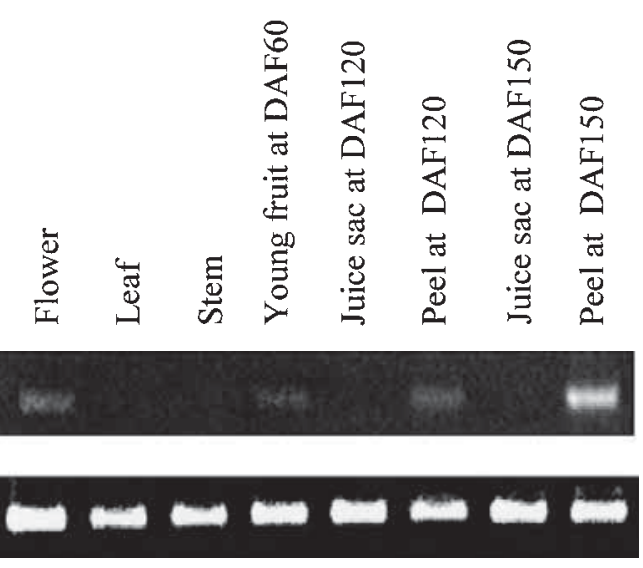

Fig. 4. Gene expression analysis of limonene synthase 1 (PtLim 1$)$ in various tissues of wild-type trifoliate orange by RT-PCR. EF1$\alpha$ was used as the control. 
(a) Transgenic leaf

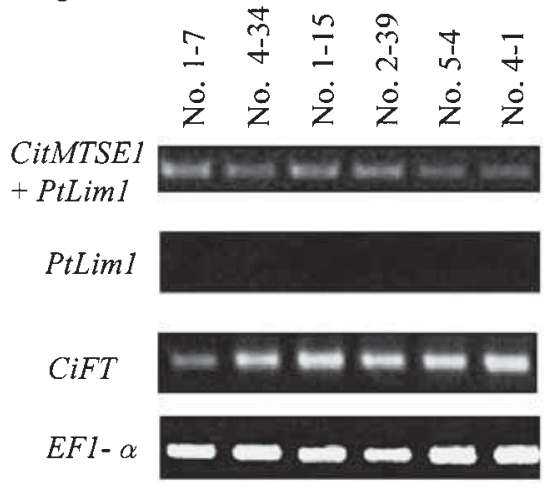

(b) Transgenic flower

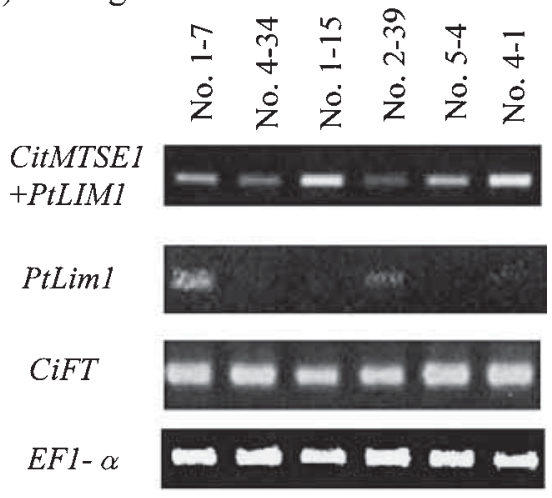

(c) Transgenic fruit

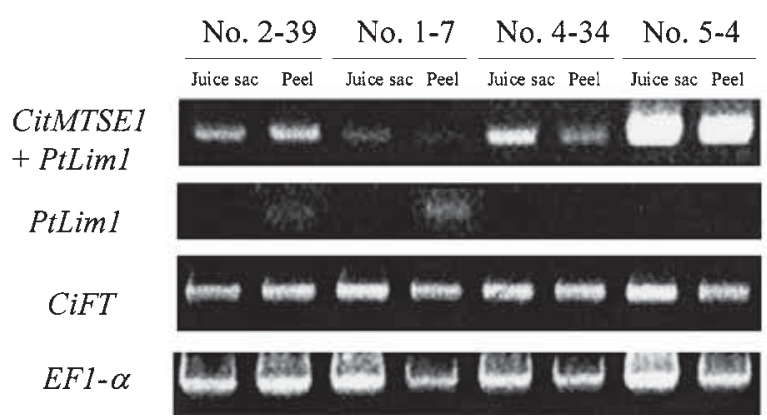

Fig. 5. Gene expression analysis of CiFT, total limonene synthase genes (introduced CitMTSE1 and endogenous PtLim1) and PtLim1 in the leaf (a), flower (b), and fruit (c) of transgenic trifoliate orange by RT-PCR. EF1- $\alpha$ was used as the control.

\section{GC-MS analysis of transgenic plants}

The flowers and peel of mature fruit from transgenic plants were analyzed using GC-MS to determine the composition of volatile components. P35S::CiFT (CNT; Endo et al., 2005) and wild-type trifoliate orange (WT) were used as controls. In flowers and peel of CNT and $\mathrm{WT}$, the major monoterpenes were $\beta$-myrcene, $d$ limonene, and (E)- $\beta$-ocimene (Fig. 6 and Table 2). Therefore, the levels of these three monoterpenes were investigated in transgenic plants. In WT flowers, the peak area percentages of $\beta$-myrcene, $d$-limonene, and (E)- $\beta$-ocimene were $29.06,26.05$, and $17.07 \%$, respectively (Table 2), which were similar to those reported previously for flowers and fruit of trifoliate orange (Sawamura, 2000). In flowers of CNT, the peak area percentages of $d$-limonene and $\beta$-myrcene were higher than in the WT, but the ratio of $d$-limonene/ $\beta$ myrcene was similar to that of the WT. Flowers of the transgenic line No. 1-7 showed values of the three major monoterpenes similar to CNT. Transgenic line Nos. 1$15,4-1,4-34$, and 5-4 showed reduced ratios of $d-$ limonene/ $\beta$-myrcene in their flowers (approximately 0.1 to 0.4). Transgenic line No. 2-39 showed an intermediate ratio of $d$-limonene/ $\beta$-myrcene in flowers. In the peel of $\mathrm{CNT}$ and WT, the ratio of $(E)-\beta$-ocimene $/ \beta$-myrcene was low compared to that in the flowers. A difference in the $(E)$ - $\beta$-ocimene content was also observed in satsuma mandarin, where $(E)$ - $\beta$-ocimene was comparatively abundant in flowers and then decreased as the fruit developed (Shimada et al., 2005a). This result was in accordance with the transcriptional repression of CitMTSL4 in fruit. Since mature fruit of transgenic line Nos. 1-15 and 4-1 were not available, the remaining four lines were analyzed. In peel of transgenic line Nos. 1-7 and 2-39, values of the three monoterpenes was similar to those in CNT and WT. In contrast, the ratio of $d$ limonene/ $\beta$-myrcene in fruit was successfully reduced to approximately 0.4 in transgenic line Nos. 4-34 and 5-4. The content of other monoterpenes, except limonene, was not significantly different in any transgenic lines, both in the flowers and fruit, when compared to CNT or the WT (data not shown).

The relative limonene content in transgenic flowers and fruit was associated with the accumulation of endogenous PtLim 1 transcripts in each line (Fig. 5b, 5c, and Table 2). Flowers and peel of line No. 1-7 accumulated transcripts of PtLiml and had a similar limonene content relative to controls. Transgenic plant Nos. 1-15, 4-34, and 5-4, all of which showed repressed endogenous PtLim 1 expression, had a reduced limonene 
(a) Transgenic flower
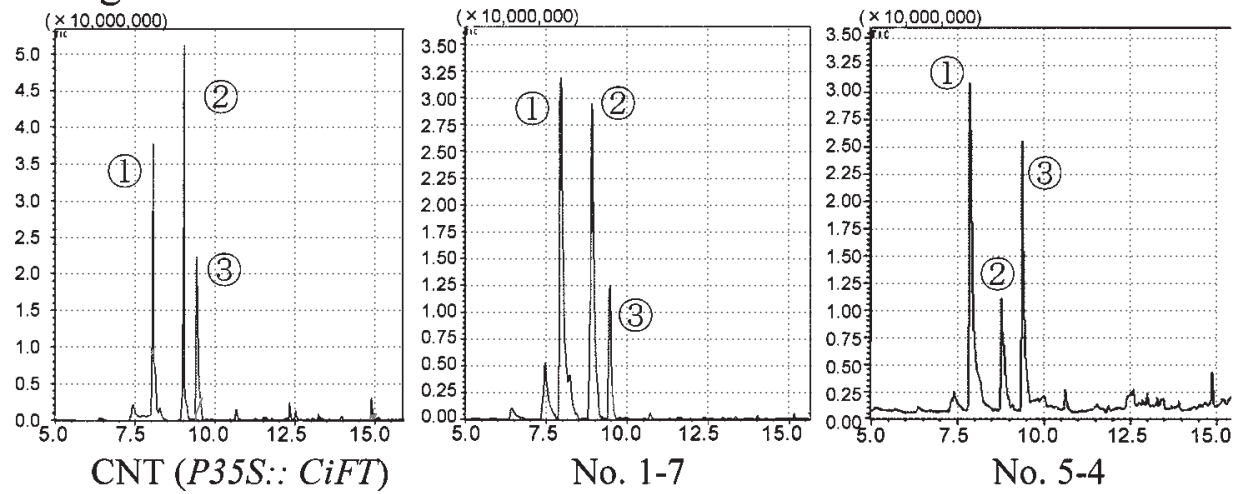

(b) Transgenic fruit
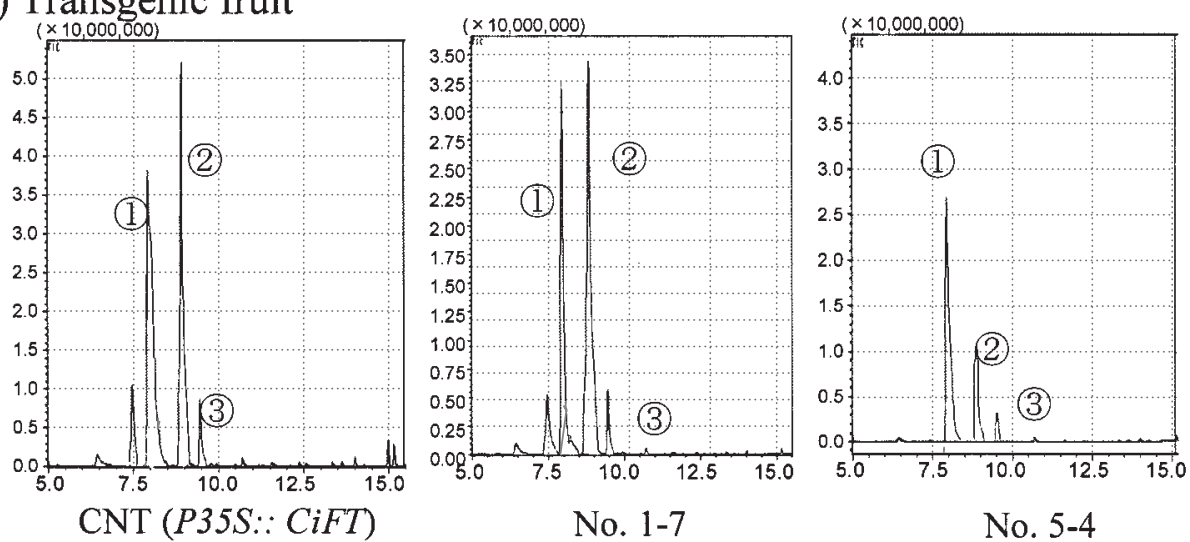

Fig. 6. GC-MS charts of aroma components in flowers (a), and fruit (b) of transgenic trifoliate orange and controls. Numbers in charts indicate

(1) $\beta$-myrcene; (2) $d$-limonene; (3) (E)- $\beta$-ocimene. Relative $d$-limonene contents in flowers and fruit of transgenic line No. 5 -4 were markedly lower than those in CNT (P35S: CiFT) and transgenic line No. 1-7.

Table 2. Peak area percentages of $\beta$-myrcene, $d$-limonene, and $(E)-\beta$-ocimene in flowers and fruit of transgenic plants and controls.

\begin{tabular}{|c|c|c|c|c|c|}
\hline & Plant material & $\beta$-myrcene $(\mathrm{Rt}=7.95)$ & $d$-limonene $(\mathrm{Rt}=8.95)$ & $(E)$ - $\beta$-ocimene $(\mathrm{Rt}=9.46)$ & \\
\hline \multirow[t]{9}{*}{ Flower } & & & Peak area $(\%)$ & & Total \\
\hline & $1-7$ & $35.5(1.00)$ & $33.08(0.93)$ & $15.32(0.43)$ & 83.9 \\
\hline & $1-15$ & $37.07(1.00)$ & $3.45(0.09)$ & $21.82(0.59)$ & 62.34 \\
\hline & $2-39$ & $34.23(1.00)$ & $24.07(0.70)$ & $17.41(0.51)$ & 75.71 \\
\hline & $4-1$ & $33.63(1.00)$ & $10.56(0.31)$ & $19.75(0.59)$ & 63.94 \\
\hline & $4-34$ & $35.72(1.00)$ & $10.77(0.30)$ & $19.21(0.54)$ & 65.7 \\
\hline & $5-4$ & $36.27(1.00)$ & $15.84(0.44)$ & $25.7 \quad(0.71)$ & 77.81 \\
\hline & P. trifoliata (WT) & $29.06(1.00)$ & $26.05(0.90)$ & $17.07(0.59)$ & 72.18 \\
\hline & $P 35 S:: C i F T(\mathrm{CNT})$ & $34.58(1.00)$ & $38.21(0.94)$ & $14.26(0.41)$ & 87.05 \\
\hline \multirow[t]{7}{*}{ Peel } & & & Peak area $(\%)$ & & Total \\
\hline & $1-7$ & $37.9(1.00)$ & $36.91(0.97)$ & $3.58(0.09)$ & 78.39 \\
\hline & $2-39$ & $30.5 \quad(1.00)$ & $33.51(1.10)$ & $2.97(0.10)$ & 66.98 \\
\hline & $4-34$ & $44.25(1.00)$ & $16.35(0.37)$ & $2.32(0.05)$ & 62.92 \\
\hline & $5-4$ & $46.1 \quad(1.00)$ & $20.06(0.44)$ & $3.41(0.07)$ & 69.57 \\
\hline & P. trifoliata (WT) & $40.25(1.00)$ & $39.74(0.99)$ & $7.38(0.18)$ & 87.37 \\
\hline & $P 35 S: \because C i F T(\mathrm{CNT})$ & $36.74(1.00)$ & $34.55(0.94)$ & $4.66(0.13)$ & 75.95 \\
\hline
\end{tabular}

Values are averages of two experimental replications.

Numbers in parentheses indicate the ratio to peak area of $\beta$-myrcene.

content in their flowers. A reduced limonene content was also observed in the fruit of Nos. 4-34 and 5-4. Fruit of No. 1-15 were not analyzed. In line No. 2-39, PtLim 1 transcripts were detected in flowers and fruit, and the relative limonene content was similar to controls in fruit. However, in flowers, the ratio of limonene was reduced 
to some extent. In this case, the transcript accumulation level in flowers of line No. 2-39 was slightly lower than that of line No. 1-7, and this repression of PtLim 1 expression was attributed to a small reduction in the relative limonene content. Similarly, it is plausible that flowers of line No. 4-1 also showed a lower accumulation of the transcript, which resulted in the reduced limonene ratio. That is, the repression of endogenous limonene synthase gene expression, which was probably caused by the ectopic expression of antisense CitMTSE1, was consistent with the reduction of the relative $d$-limonene content.

\section{Discussion}

Our results clearly indicated that the CiFT coexpression system could reduce the time to obtain flowers and fruit of transgenic trifoliate orange, thus enabling the rapid evaluation of the effects of gene introduction into reproductive tissues. Indeed, this is the first successful alteration of metabolic components in flowers and fruit of transgenic citrus plants. The long juvenile period of citrus, ranging from 6 to 20 years, has hampered the genetic improvement of reproductive tissues in transgenic plants. Although there have been some reports on gene introduction aimed at the improvement of citrus fruit quality (Guo et al., 2005; Li et al., 2002), only vegetative tissues in transgenic plants have been investigated so far. The introduction of the CiFT co-expression vector caused early flowering and fruiting in transgenic trifoliate orange. Transgenic plants producing flowers and fruit were obtained within 2 years of Agrobacterium infection, which is almost the same as that reported previously (Endo et al., 2005). Although all 14 transgenic lines obtained in this study flowered, fruit development was observed only in 6 lines within 2 years of Agrobacterium infection. Since most of plants without fruiting had a dwarfed and heavily branched tree shape and small leaves, similar observations to a previous report (Endo et al., 2005), nutritional problems might prevent fruit development in these transgenic plants. The frequent obtaining of fruit-bearing transgenic plants is important to improve this system. This system can be used to alter expression of the target gene, limonene synthase, in reproductive organs of flowers and fruit, resulting in a significant reduction in the relative limonene content in transgenic plants. Therefore, this system will make a useful contribution to advancing the metabolic engineering of citrus. There have been several attempts to alter plant aroma using monoterpene synthase genes. Transgenic tobacco that over-expressed monoterpene synthase genes showed a small accumulation of their corresponding products, or products that were inactivated by glycosylation or hydroxylation (Lücker et al., 2004a, 2004b; Ohara et al., 2003). Transgenic tomato that expressed the geraniol synthase (GES) gene in mature fruit showed marked changes in the composition of volatiles, along with unexpected increases of myrcene, limonene, and ocimene (Davidovich-Rikanati et al., 2007). In citrus, peels are especially rich in oil glands containing essential oils. These essential oils consist of various terpenoids, such as hemiterpenes, monoterpenes, and sesquiterpenes, and their derivatives, such as alcohols, esters, and acetates. They are known to be involved in ecological interactions with insects, pathogens, and other plants and are commercially valuable in terms of fragrances, pharmaceuticals, and agrochemicals. For example, $d$-limonene and 1,8-cineole have insecticide and anti-fungal activities (Byun-McKay et al., 2006; Pattnaik et al., 1997). Since this study showed that endogenous monoterpene metabolism in citrus fruit can be modified in transgenic plants, our approach will potentially lead to new insights into the biological functions of various monoterpenes and the regulation of monoterpene metabolism.

In transgenic plants, the reduced levels of $d$-limonene in flowers and fruit were consistent with the transcriptional repression of the endogenous gene. This suggests that monoterpene biosynthesis is regulated at a transcriptional level. The same hypothesis was generated by previous results, in that a different volatile composition in each tissue was explained by transcriptional accumulation of the corresponding monoterpene synthase genes (Shimada et al., 2005a). It was reported that limonene is the predominant component of citrus aroma, since the $\mathrm{Km}$ value of limonene synthase was 4.5 -fold lower than the $\mathrm{Km}$ values of $\beta$-pinene and $\gamma$ terpinene synthases (Lücker et al., 2002). However, in this study, the composition of other monoterpenes was not significantly altered in transgenic plants in spite of the reduction of the transcripts for the endogenous limonene synthase gene and relative $d$-limonene level. Limonene synthase forms $d$-limonene directly from geranyl pyrophosphate (GPP), a common $\mathrm{C}_{10}$ intermediate of isoprenoid biosynthesis, through the initial isomerization of GPP to the tertiary allylic isomer, linalyl pyrophosphate, and subsequent cyclization of this intermediate to the $\alpha$-terpinyl carbocation (Fig. 7, McGarvey and Croteau, 1995). GPP is generated by the condensation of isopentenyl diphosphate (IPP) and its allylic isomer dimethylallyl diphosphate (DMAPP) in the plastidial 2-C-methyl-D-erythritol 4-phosphate (MEP) pathway (Fig. 7, Rodriguez-Concepción and Boronat, 2002). IPP and DMAPP in the plastidial MEP pathway are responsible for the production of isoprene (C5), monoterpenes, diterpenes (C20; e.g., gibberellins), and tetraterpenes (C40; e.g., carotenoids). Therefore, we assumed that the GPP or IPP pool in transgenic flowers and fruit was consumed by other biosynthesis pathways, such as that involving gibberellins (van Schie et al., 2007), and the transcriptional reduction of the limonene synthase gene did not lead to a significant change in the levels of other monoterpenes.

In conclusion, the CiFT co-expression system is useful for the rapid evaluation of transgenic flowers and fruit, 


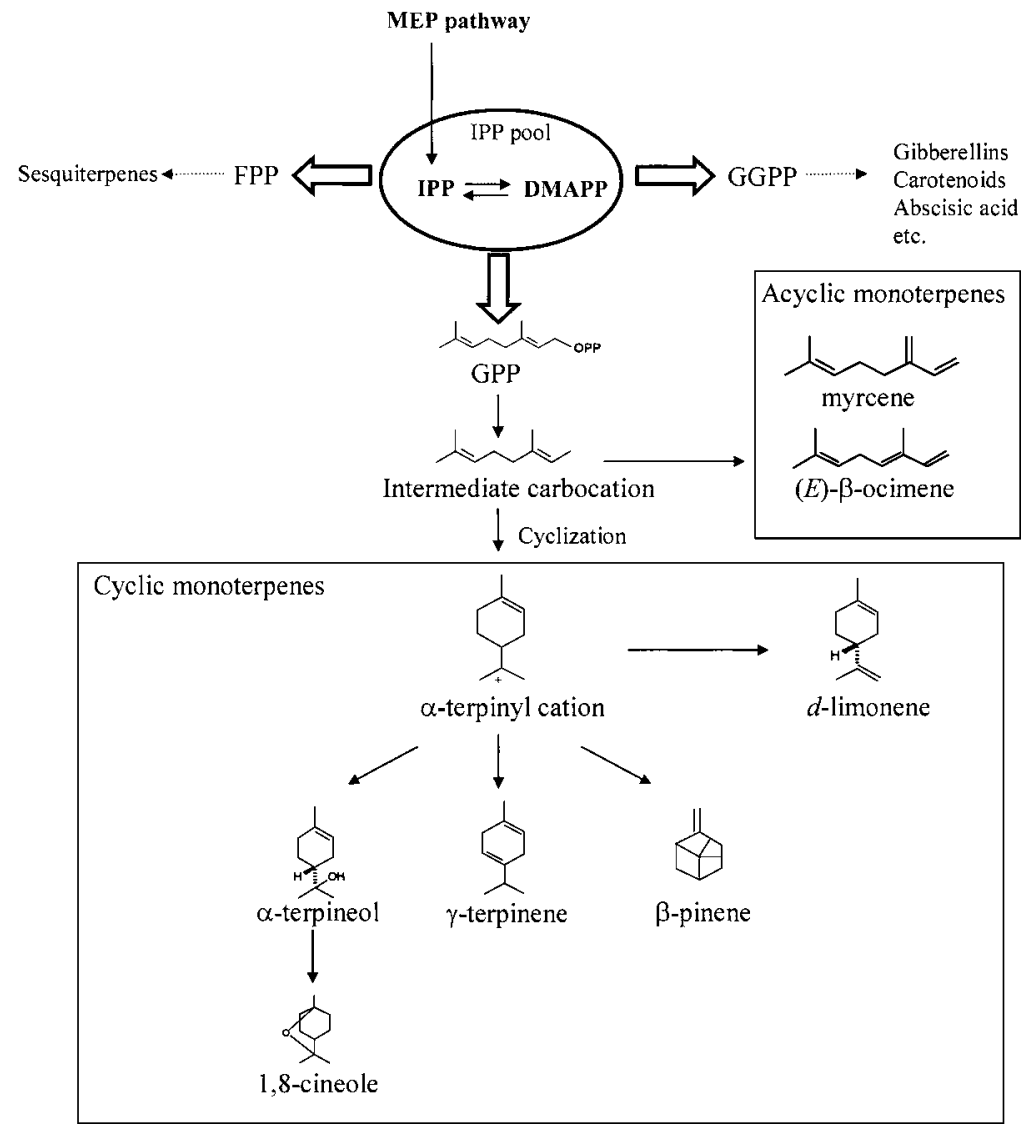

Fig. 7. Schematic diagram of the biosynthesis of monoterpenes discussed in this experiment. Isopentenyl pyrophosphate (IPP) and its allylic isomer dimethylallyl diphosphate (DMAPP) are used for the synthesis of GPP, FPP, and GGPP, which are precursors of higher terpenoids such as monoterpenes, sesquiterpenes, carotenoids etc. The formation of monoterpenes occurs through the intermediate carbocation. The decisive difference in the biosynthesis of acyclic and cyclic monotepenes depends on the cyclization of the intermediate carbocation.

and to study metabolic improvement in citrus. Citrus fruit is abundant in secondary metabolites, in particular, various terpenoids such as monoterpenes, sesquiterpenes, limonoids, and carotenoids, some of which could be utilized in pharmaceuticals and agrochemicals. Several studies involving citrus have suggested that the levels of secondary metabolites were directly affected by their transcriptional levels (Jacob-Wilk et al., 1999; Kato et al., 2004; Shimada et al., 2005a). The CiFT coexpression system can be applied to such secondary metabolites, and, thus, advance metabolic engineering. Recently, citrus promoters were isolated from satsuma mandarin and characterized, such as CuMFT1 (Nishikawa et al., 2008) and CitMT45 (Endo et al., 2007) promoters, which induce preferential expressions in seeds and fruit, respectively. The use of these promoters in the CiFT co-expression system will advance citrus transgenic studies, and contribute to the production of commercially desirable transgenic plants for use in pharmaceutical and agrochemical industries.

\section{Acknowledgements}

We are indebted to Ms. Misako Ikeda, Ms. Akemi Aoki, and Ms. Yoko Makita for their technical assistance.
This study was supported by the Program for the Promotion of Basic Research Activities for Innovative Biosciences (PROBRAIN).

\section{Literature Cited}

Abe, M., Y. Kobayashi, S. Yamamoto, Y. Daimon, A. Yamaguchi, Y. Ikeda, H. Ichinoki, M. Notaguchi, K. Goto and T. Araki. 2005. FD, a bZIP protein mediating signals from the floral pathway integrator FT at the shoot apex. Science 309: 10521056.

Byun-McKay, A., K. A. Godard, M. Toudefallah, D. M. Martin, R. Alfaro, J. King, J. Bohlmann and A. L. Plant. 2006. Woundinduced terpene synthase gene expression in Sitka spruce that exhibit resistance or susceptibility to attack by the white pine weevil. Plant Physiol. 140: 1009-1021.

Corbesier, L., C. Vincent, S. Jang, F. Fornara, Q. Fan, I. Searle, A. Giakountis, S. Farrona, L. Gissot, C. Turnbull and G. Coupland. 2007. FT protein movement contributes to longdistance signaling in floral induction of Arabidopsis. Science 316: 1030-1033.

Davidovich-Rikanati, R., Y. Sitrit, Y. Tadmor, Y. Iijima, N. Bilenko, E. Bar, B. Carmona, E. Fallik, N. Dudai, J. E. Simon, E. Pichersky and E. Lewinsohn. 2007. Enrichment of tomato flavor by diversion of the early plastidial terpenoid pathway. Nat. Biotech. 25: 899-901.

Dellaporta, S. L., J. Wood and J. B. Hicks. 1983. A plant DNA 
minipreparation: version II. Plant Mol. Biol. Rep. 4: 19-21.

Endo, T., T. Shimada, H. Fujii, Y. Kobayashi, T. Araki and M. Omura. 2005. Ectopic expression of an $F T$ homolog from Citrus confers an early flowering phenotype on trifoliate orange (Poncirus trifoliata L. Raf.). Transgenic Res. 14: 703 712 .

Endo, T., T. Shimada, H. Fujii, T. Moriguchi and M. Omura. 2007. Promoter analysis of a type 3 metallothionein-like gene abundant in Satsuma mandarin (Citrus unshiu Marc.) fruit. Sci. Hort. 112: 207-214.

Guo, W., Y. Duan, O. Olivares-Fuster, Z. Wu, C. R. Arias, J. K. Burns and J. W. Grosser. 2005. Protoplast transformation and regeneration of transgenic Valencia sweet orange plants containing a juice quality-related pectin methylesterase gene. Plant Cell Rep. 24: 482-486.

Hara, M., M. Kishimoto and T. Kuboi. 1999. Changes of $d$ limonene content in three Citrus species during fruit development. Food Sci. Technol. Res. 5: 80-81.

Ikoma, Y., M. Yano, K. Ogawa, T. Yoshioka, Z. C. Xu, S. Hisada, M. Omura and T. Moriguchi. 1996. Isolation and evaluation of RNA from polysaccharide-rich tissues in fruit for quality by cDNA library construction and RT-PCR. J. Japan. Soc. Hort. Sci. 64: 809-814.

Jacob-Wilk, D., D. Holland, E. E. Goldschmidt, J. Riov and Y. Eyal. 1999. Chlorophyll breakdown by chlorophyllase: isolation and functional expression of the Chlase 1 gene from ethylene-treated Citrus fruit and its regulation during development. Plant J. 20: 653-661.

Kato, M., Y. Ikoma, H. Matsumoto, M. Sugiura, H. Hyodo and M. Yano. 2004. Accumulation of carotenoids and expression of carotenoid biosynthetic genes during maturation in citrus fruit. Plant Physiol. 134: 824-837.

Kobayashi, Y., H. Kaya, K. Goto, M. Iwabuchi and T. Araki. 1999. A pair of related genes with antagonistic roles in mediating flowering signals. Science 286: 1960-1962.

Lewinsohn, E., F. Schalechet, J. Wilkinson, K. Matsui, Y. Tadmor, K. H. Nam, O. Amar, E. Lastochkin, O. Larkov, U. Ravid, W. Hiatt, S. Gepstein and E. Pichersky. 2001. Enhanced levels of the aroma and flavor compound S-linalool by metabolic engineering of the terpenoid pathway in tomato fruits. Plant Physiol. 127: 1256-1265.

Li, D. D., W. Shi and X. X. Deng. 2002. Agrobacterium-mediated transformation of embryogenic calluses of Ponkan mandarin and the regeneration of plants containing the chimeric ribonuclease gene. Plant Cell Rep. 21: 153-156.

Loza-Tavera, H. 1999. Monoterpenes in essential oils-Biosynthesis and properties. Adv. Exp. Med. Biol. 464: 49-62.

Lücker, J., H. J. Bouwmeester, W. Schwab, J. Blaas, L. H. van der Plas and H. A. Verhoeven. 2001. Expression of Clarkia $S$-linalool synthase in transgenic petunia plants results in the accumulation of $S$-linalyl- $\beta$-D-glucopyranoside. Plant J. 27: 315-324.

Lücker, J., M. K. El Tamer, W. Schwab, F. W. A. Verstappen, L. H. W. van der Plas, H. J. Bouwmeester and H. A. Verhoeven. 2002. Monoterpene biosynthesis in lemon (Citrus limon): cDNA isolation and functional analysis of four monoterpene synthases. Eur. J. Biochem. 269: 3160-3171.

Lücker, J., W. Schwab, M. C. Franssen, L. H. van der Plas, H. J. Bouwmeester and H. A. Verhoeven. 2004a. Metabolic engineering of monoterpene biosynthesis: two-step production of (+)-trans-isopiperitenol by tobacco. Plant J. 39: 135145.

Lücker, J., W. Schwab, B. van Hautum, J. Blaas, L. H. W. van der Plas, H. J. Bouwmeester and H. A. Verhoeven. 2004b.
Increased and altered fragrance of tobacco plants after metabolic engineering using three monoterpene synthases from lemon. Plant Physiol. 134: 510-519.

McGarvey, D. J. and R. Croteau. 1995. Terpenoid metabolism. Plant Cell 7: 1015-1026.

Mitsuhara, I., M. Ugaki, H. Hirochika, M. Ohshima, T. Murakami, Y. Gotoh, Y. Katayose, S. Nakamura, R. Honkura, S. Nishimiya, K. Ueno, A. Mochizuki, H. Tanimoto, H. Tsugawa, Y. Otsuki and Y. Ohashi. 1996. Efficient promoter cassettes for enhanced expression of foreign genes in dicotyledonous and monocotyledonous plants. Plant Cell Physiol. 37: 49-59.

Nishikawa, F., T. Endo, T. Shimada, H. Fujii, T. Shimizu and M. Omura. 2008. Isolation and characterization of a Citrus FT/ TFL1 homologue (CuMFT1), which shows quantitatively preferential expression in Citrus seeds. J. Japan. Soc. Hort. Sci. 77: 38-46.

Ohara, K., T. Ujihara, T. Endo, F. Sato and K. Yazaki. 2003. Limonene production in tobacco with Perilla limonene synthase cDNA. J. Exp. Bot. 54: 2635-2642.

Pattnaik, S., V. R. Subramanyam, M. Bapaji and C. R. Kole. 1997. Antibacterial and antifungal activity of aromatic constituents of essential oils. Microbios 89: 39-46.

Peña, L., M. Martín-Trillo, J. Juárez, J. A. Pina, L. Navarro and J. M. Martínez-Zapater. 2001. Constitutive expression of Arabidopsis LEAFY or APETALA1 genes in citrus reduces their generation time. Nat. Biotech. 19: 263-267.

Rodríguez-Concepción, M. and A. Boronat. 2002. Elucidation of the methylerythritol phosphate pathway for isoprenoid biosynthesis in bacteria and plastids. A metabolic milestone achieved through genomics. Plant Physiol. 130: 1079-1089.

Sawamura, M. 2000. Volatile components of essential oils of the Citrus genus. p. 131-164. In: S. G. Pandali (ed.). Recent Research Developments in Agricultural and Food Chemistry. Vol. 4. Research Signpost, Trivandrum, India.

van Schie, C. C., K. Ament, A. Schmidt, T. Lange, M. A. Haring and R. C. Schuurink. 2007. Geranyl diphosphate synthase is required for biosynthesis of gibberellins. Plant J. 52: 752-762.

Shimada, T., T. Endo, H. Fujii, M. Hara, T. Ueda, M. Kita and M. Omura. 2004. Molecular cloning and functional characterization of four monoterpene synthase genes from Citrus unshiu Marc. Plant Sci. 166: 49-58.

Shimada, T., T. Endo, H. Fujii, M. Hara and M. Omura. 2005a. Isolation and characterization of $(E)$ - $\beta$-ocimene and 1,8 cineole synthases in Citrus unshiu Marc. Plant Sci. 168: 987 995.

Shimada, T., T. Endo, H. Fujii and M. Omura. 2005b. Isolation and characterization of a new $d$-limonene synthase gene with a different expression pattern in Citrus unshiu Marc. Sci. Hort. 105: 507-512.

Takada, S. and K. Goto. 2003. TERMINAL FLOWER2, an Arabidopsis homolog of HETEROCHROMATIN PROTEIN1, counteracts the activation of FLOWERING LOCUS T by CONSTANS in the vascular tissues of leaves to regulate flowering time. Plant Cell 15: 2856-2865.

Tamaki, S., S. Matsuo, H. L. Wong, S. Yokoi and K. Shimamoto. 2007. Hd3a protein is a mobile flowering signal in rice. Science 316: 1033-1036.

Yanisch-Perron, C., J. Vieira and J. Messing. 1985. Improved M13 phage cloning vectors and host strains: nucleotide sequences of the M13mp18 and pUC19 vectors. Gene 33: 103-119.

Yoshida, T., H. Nesumi, T. Yoshioka, M. Nakano, Y. Ito, S. Murase and F. Takishita. 2005. New citrus cultivar 'Harehime'. Bull. Natl. Inst. Fruit Tree Sci. 4: 37-45. 